\title{
Equivalence between Modulus of Smoothness and K-Functional on Rotation Group SO(3)*
}

\author{
Zhuyuan Yang1, Zongwen Yang² \\ ${ }^{1}$ School of Mathematics and computer Science, Yunnan Minzu University, Kunming, China \\ ${ }^{2}$ Department of Mathematics, Yunnan University, Kunming, China \\ Email:1025719371@qq.com,yangzhuyuan@sina.com,zwyang@ynu.edu.cn
}

How to cite this paper: Yang, Z.Y. and Yang, Z.W. (2017) Equivalence between Modulus of Smoothness and K-Functional on Rotation Group $\mathrm{SO}(3)$. Journal of Applied Mathematics and Physics, 5, 341-345. https://doi.org/10.4236/jamp.2017.52031

Received: January 18, 2017

Accepted: February 12, 2017

Published: February 15, 2017

\begin{abstract}
In this paper we obtain the equivalence between modulus of smoothness and K-functional on rotation group $\mathrm{SO}(3)$.
\end{abstract}

\section{Keywords}

Rotation Group, Modulus of Smoothness, K-Functional, Equivalence

\section{Introduction}

Many results of approximation are based on Euclid spaces or their compact subsets. Periodic approximation is based on compact group $\{\exp (i x)\}$, whereas matrix group $U(n)$ is the generalization of $\{\exp (i x)\}$. We know homomorphism between $\mathrm{SU}(2)$ and rotation group $\mathrm{SO}(3)$, which has many applications in Physics and Chemistry. Some approximation problems on compact groups have been studied since in 1920s F. Peter and H. Weyl proved the approximation theorem on compact group, that is, the irreducible character generate a dense subspace of the space of continuous classes function. For instance, Gongsheng (see [1]) studied the basic problems of Fourier analysis on unitary and rotation groups, including the degree of convergence of Abel sum based on Poisson kernel. Xue-an Zheng (see [2] [3]) studied the polynomial approximation on compact Lie groups. D. I. Cartwright et al. studied Jackson's theorem for compact connected Lie groups (see [4]), and so on. In this paper, we study the modulus of smoothness and $\mathrm{K}$-functional on rotation group $\mathrm{SO}(3)$ and as classical casein Euclid space we will obtain the equivalence between them.

Let $G=S O(3)=\left\{\mathbf{x} \in G L(3 ; \mathbb{R}) ; \mathbf{x}^{\mathrm{T}} \mathbf{x}=\mathbf{E} ; \operatorname{det} \mathbf{x}=1\right\}$ be the rotation group, where $G L(n, \mathbb{R})$ is the group of invertible real $(n \times n)$ matrices. For $1 \leq p<+\infty$,

${ }^{\star}$ This research was supported by the national natural science foundation of China (11361076). 
$L_{p}(G)\left\{f:\left[\int_{G}|f(x)|^{p} \mathrm{~d} \mu(x)\right]^{1 / p}<+\infty\right\}$, where $\mu$ is the normalized Harr measure on $G$. For $D_{i} \in \mathbf{g}$, the Lie algebra of $G=S O(3), i=1,2,3$, Let

$L_{p, i}^{r}(G)=\left\{f: D_{i}^{r} f \in L_{p}(G)\right\}, i=1,2,3$, where $D_{i}^{r} g$ denote the r-order derivative of $\mathrm{g}$ in direction $D_{i}$.

We also write the difference of function $f$ and modulus of smoothness in the direction $D_{i}$ as follows

$$
\Delta_{s D_{i}}^{r} f(x)=\sum_{j=0}^{r}(-1)^{r-j}\left(\begin{array}{l}
r \\
j
\end{array}\right) f\left(x \exp j s D_{i}\right),
$$

and

$$
\omega_{r, i}(f, \delta)_{p}=\sup \left\{\left\|\Delta_{s D_{i}}^{r} f\right\|_{p}: 0<s<\delta,\left\|D_{i}\right\|=1\right\}
$$

where $\left\|D_{i}\right\|$ is the norm induced by Killing inner product on $\mathbf{g}$.

We denote

$$
W_{r}(f, \delta)_{p}=\sum_{i=1}^{3} \omega_{r, i}(f, \delta)_{p} .
$$

Accordingly, we denote K-functional as follows

$$
\begin{gathered}
K_{r, i}(f, \delta)_{p}=\inf _{g \in L_{p, i}^{r}(G)}\left\{\|f-g\|_{p}+\delta^{r}\left\|D_{i}^{r} g\right\|_{p}\right\} \\
K_{r}(f, \delta)_{p}=\sum_{i=1}^{3} K_{r, i}(f, \delta)_{p}
\end{gathered}
$$

Further, for the isotropic case.

Let multi-indice $\mathbf{r}=\left(r_{1}, r_{2}, r_{3}\right) \in N_{+}^{3}$, and $\mathbf{s}=\left(s_{1}, s_{2}, s_{3}\right)>\mathbf{0}, \mathbf{t}=\left(t_{1}, t_{2}, t_{3}\right)$, $\Lambda=\left\{\mathbf{a}=\left(\alpha_{1}, \alpha_{2}, \alpha_{3}\right): \mathbf{a}<\mathbf{r}\right\}, \partial \Lambda=\left\{r_{i} e_{i}, i=1,2,3\right\}$, here $e_{i}$ is the unit vector in the $i$-th direction. Define

$$
\Delta_{s}^{r} f(x)=\Delta_{s_{1} D_{1}}^{r_{1}} \Delta_{s_{2} D_{2}}^{r_{2}} \Delta_{s_{3} D_{3}}^{r_{3}} f(x),
$$

and

$$
\omega_{\mathbf{r}}(f, \mathbf{t})_{p}=\sup _{0<\mathbf{s}<\mathbf{t}}\left\|\Delta_{\mathrm{s}}^{\mathrm{r}} f\right\|_{p}=\sup \left\{\left\|\Delta_{s_{1} D_{1}}^{r_{1}} \Delta_{s_{2} D_{2}}^{r_{2}} \Delta_{s_{3} D_{3}}^{r_{3}} f\right\|_{p}: \mathbf{0}<\mathbf{s}<\mathbf{t},\left\|D_{i}\right\|=1, i=1,2,3\right\},
$$

and

$$
W_{\Lambda}(f, \mathbf{t})_{p}=\sum_{\mathbf{r} \in \partial \Lambda} \omega_{\mathbf{r}}(f, \mathbf{t})_{p} .
$$

The corresponding $K$-functional is defined by

$$
K_{\Lambda}(f, \mathbf{t})_{p}=\inf _{g \in L_{p}^{\Lambda}}\left\{\|f-g\|_{p}+\sum_{\mathbf{r} \in \partial \Lambda} \mathbf{t}^{\mathbf{r}}\left\|\mathbf{D}^{\mathbf{r}} g\right\|_{p}\right\},
$$

where $L_{p}^{\Lambda}=L_{p}^{\Lambda}(G)=\left\{f: \mathbf{D}^{\mathbf{r}} f \in L_{p}(G), \mathbf{r} \in \partial \Lambda\right\}, \quad \mathbf{t}^{\mathbf{r}}=t_{1}^{r_{1}} t_{2}^{r_{2}} t_{3}^{r_{3}}, \quad \mathbf{D}^{\mathbf{r}}=D_{1}^{r_{1}} D_{2}^{r_{2}} D_{3}^{r_{3}}$.

In the next paragraph we denote by $C$ or $C_{i}$ the positive constants but are not the same in the different formula. And $A \asymp B$ means there exist two positive constants $C_{1}, C_{2}$ satisfying $C_{1} A \leq B \leq C_{2} A$.

\section{Theorems and Their Proofs}

We will use the next lemma 1. 
Lemma 1 [5] [6]. If $f \in L_{1}^{r}[t, t+s]$, then $\Delta_{s}^{r} f(t)=s^{r} \int_{0}^{r s} \frac{f^{(r)}(t+u) N_{r}(u / s)}{s} \mathrm{~d} u$, where $N_{r}$ denotes the normalized B-spline of order $r$ (degree $r-1)$.

Theorem 1. If $f \in L_{p}(S O(3)), 1 \leq p<+\infty, r \in \mathbb{N}_{+}, 0<\delta \in \mathbb{R}$, then

$$
W_{r}(f, \delta)_{p} \asymp K_{r}(f, \delta)_{p} .
$$

Proof. For $i=1,2,3$, we first construct the approximation operators as follows

$$
\begin{aligned}
g_{i}(x) & =g_{s D_{i}}(x)=I_{i} f(x) \\
& =\int_{0}^{r s}\left[f(x)+(-1)^{r-1} \Delta_{u D_{i}}^{r} f(x)\right] \frac{N_{r}(u / s)}{s} \mathrm{~d} u \\
& =\int_{0}^{r s} \sum_{j=1}^{r}(-1)^{j+1}\left(\begin{array}{l}
r \\
j
\end{array}\right) f\left(x \exp \left(j u D_{i}\right)\right) \frac{N_{r}(u / s)}{s} \mathrm{~d} u \\
& =\sum_{j=1}^{r}(-1)^{j+1}\left(\begin{array}{c}
r \\
j
\end{array}\right) \int_{0}^{r s} f\left(x \exp \left(j u D_{i}\right)\right) \frac{N_{r}(u / s)}{s} \mathrm{~d} u \\
& =\sum_{j=1}^{r}(-1)^{j+1}\left(\begin{array}{c}
r \\
j
\end{array}\right) \int_{0}^{j r s} f\left(x \exp \left(t D_{i}\right)\right) \frac{N_{r}(t / j s)}{j s} \mathrm{~d} t .
\end{aligned}
$$

By Lemma 1,

$$
g_{i}(x)=\sum_{j=1}^{r}(-1)^{j+1}\left(\begin{array}{l}
r \\
j
\end{array}\right)(j s)^{-r} \Delta_{j s D_{i}}^{r} F(x)
$$

where $D^{r} F=f$.

Obviously, $I_{i}$ is a bounded operator from $L_{p}$ to $L_{p}(1 \leq p<+\infty)$.

If we differentiate $r$ times, then

$$
D_{i}^{r} g_{i}(x)=\sum_{j=1}^{r}(-1)^{j+1}\left(\begin{array}{l}
r \\
j
\end{array}\right)(j s)^{-r} \Delta_{j s D_{i}}^{r} f(x),
$$

So,

$$
s^{r}\left\|D_{i}^{r} g_{i}\right\|_{p} \leq c_{r}\left\|\Delta_{s D_{i}}^{r} f\right\|_{p} \leq c_{r} \omega_{r, i}(f, s)_{p} .
$$

Clearly,

$$
\left\|f-g_{i}\right\|_{p} \leq C \omega_{r, i}(f, s)_{p} .
$$

We get

$$
K_{r, i}(f, s)_{p} \leq C \omega_{r, i}(f, s)_{p},
$$

and

$$
K_{r}(f, \delta)_{p} \leq C W_{r}(f, \delta)_{p} .
$$

Conversely, for $g_{i}(x) \in L_{p, i}(G)$, using (see [7])

$$
\Delta_{s D_{i}}^{r} f(x)=\int_{o}^{s} \mathrm{~d} t_{1} \cdots \int_{o}^{s} \mathrm{~d} t_{r} D_{i}^{r} g_{i}\left(x \exp \left(t_{1}+\cdots+t_{r}\right) D_{i}\right),
$$

we have

$$
\begin{aligned}
W_{r}(f, \delta)_{p} & =\sum_{i=1}^{3} \omega_{r, i}(f, \delta)_{p} \leq \sum_{i=1}^{3}\left[\omega_{r, i}\left(f-g_{i}, \delta\right)_{p}+\omega_{r, i}\left(g_{i}, \delta\right)_{p}\right] \\
& \leq C \sum_{i=1}^{3}\left[\left\|f-g_{i}\right\|_{p}+\delta^{r}\left\|D_{i}^{r} g_{i}\right\|_{p}\right] .
\end{aligned}
$$


Thus

$$
W_{r}(f, \delta)_{p} \leq C K_{r}(f, \delta)_{p} .
$$

Theorem 2. For $f \in L_{p}(G), 1 \leq p<+\infty, \quad \mathbf{t}>0$, then

$$
W_{\Lambda}(f, \mathbf{t})_{p} \asymp K_{\Lambda}(f, \mathbf{t})_{p} .
$$

Proof. Noting that for $g \in L_{p}^{\Lambda}$,

$$
\omega_{\mathbf{r}}(g, \mathbf{t})_{p} \leq \mathbf{t}^{\mathbf{r}}\left\|\mathbf{D}^{\mathbf{r}} g\right\|_{p},
$$

we get

$$
W_{\Lambda}(g, \mathbf{t})_{p} \leq \sum_{\mathbf{r} \in \partial \Lambda} \mathbf{t}^{\mathbf{r}}\left\|\mathbf{D}^{\mathbf{r}} g\right\|_{p}
$$

Writing $f=f-g+g$ and using the last inequality will give

$$
W_{\Lambda}(f, \mathbf{t})_{p} \leq C K_{\Lambda}(f, \mathbf{t})_{p} .
$$

Moreover, we construct the approximation operator as follows

$$
\begin{aligned}
g(x) & =I(f, x)=I_{1} I_{2} I_{3}(f, x) \\
& =\int_{0}^{r_{1} s_{1}} \int_{0}^{r_{2} s_{2}} \int_{0}^{r_{3} s_{3}}\left[f(x)+\Delta_{\mathbf{s}}^{\mathbf{r}} f(x)\right] N_{\mathbf{r}}(\mathbf{s}, \mathbf{u}) \mathrm{d} u_{1} \mathrm{~d} u_{2} \mathrm{~d} u_{3},
\end{aligned}
$$

where

$$
N_{\mathbf{r}}(\mathbf{s}, \mathbf{u})=\frac{N_{r_{1}}\left(u_{1} / s_{1}\right) N_{r_{2}}\left(u_{2} / s_{2}\right) N_{r_{3}}\left(u_{3} / s_{3}\right)}{s_{1} s_{2} s_{3}} .
$$

It easy to see that by using the boundedness of $I_{i}, i=1,2,3$.

$$
\begin{aligned}
& \|f-g\|_{p} \leq\left\|f-I_{1} f\right\|_{p}+\left\|I_{1} f-I_{1} I_{2} f\right\|_{p}+\left\|I_{1} I_{2} f-I_{1} I_{2} I_{3} f\right\|_{p} \\
& \leq C \sum_{i=1}^{3} \omega_{r, i}\left(f, t_{i}\right)_{p}=C W_{\Lambda}(f, \mathbf{t})_{p}
\end{aligned}
$$

It is similarly to (1), we have

$$
\mathbf{t}^{\mathbf{r}}\left\|D^{\mathbf{r}} g\right\|_{p} \leq\left\|\Delta_{\mathrm{s}}^{\mathbf{r}} f\right\|_{p} \text { and } \sum_{\mathbf{r} \in \partial \Lambda} \mathbf{t}^{\mathbf{r}}\left\|D^{\mathbf{r}} g\right\|_{p} \leq W_{\Lambda}(f, \mathbf{t}) .
$$

Thus $K_{\Lambda}(f, \mathbf{t})_{p} \leq C W_{\Lambda}(f, \mathbf{t})_{p}$.

Remark: Theorem 1 and theorem 2 can be easily generalized to $\mathrm{SO}(n)(n>3)$.

\section{References}

[1] Gong, S. (1983) Harmonic Analysis on Classical Groups. Science Press, Beijing. (In Chinese).

[2] Zheng, X.-A., Xu, Z.F. and Zhao, H.S. (987) Approximation by Polynomial on Compact Lie Groups(I). Advance in Mathematics, 16, 61-66. (In Chinese)

[3] Zheng, X.-A., Zhao, H.S. and Xu, Z.F. (1990) Polynomial Approximation on Compact Lie Groups in the $L_{p}$ Norm(II). Advance in Mathematics, 19, 199-203. (In Chinese).

[4] Cartwright, D.I. and Kucharski, K. (1988) Jackson's Theorem for Compact Connected Lie Groups. Journal of Approximation Theory, 55, 352-359. https://doi.org/10.1016/0021-9045(88)90102-5

[5] Schumarker, L.L. (1981) Spline Functions: Basic Theory. A Wiley-Interscience Publication, John Wiley and Sons Inc., New York, Chichester, Brisbane, Toronto. 
[6] Dahmen, W., De Vore, R. and Scherer, K. (1980) Multi-Dimensional Spline Approximation. SIAM Journal on Numerical Analysis, 17, 380-402. https://doi.org/10.1137/0717033

[7] Gaudry, G.I. and Pini, R. (1986) Bernstein Theorem for Compact, Connected Lie Groups. Mathematical Proceedings of the Cambridge Philosophical Society, 99, 297305. https://doi.org/10.1017/S0305004100064215

Submit or recommend next manuscript to SCIRP and we will provide best service for you:

Accepting pre-submission inquiries through Email, Facebook, LinkedIn, Twitter, etc. A wide selection of journals (inclusive of 9 subjects, more than 200 journals)

Providing 24-hour high-quality service

User-friendly online submission system

Fair and swift peer-review system

Efficient typesetting and proofreading procedure

Display of the result of downloads and visits, as well as the number of cited articles Maximum dissemination of your research work

Submit your manuscript at: http://papersubmission.scirp.org/

Or contact jamp@scirp.org 